\title{
Comprehensive genomic characterization of head and neck squamous cell carcinomas
}

\author{
The Cancer Genome Atlas Network*
}

The Cancer Genome Atlas profiled 279 head and neck squamous cell carcinomas (HNSCCs) to provide a comprehensive landscape of somatic genomic alterations. Here we show that human-papillomavirus-associated tumours are dominated by helical domain mutations of the oncogene PIK3CA, novel alterations involving loss of TRAF3, and amplification of the cell cycle gene E2F1. Smoking-related HNSCCs demonstrate near universal loss-of-function TP53 mutations and CDKN2A inactivation with frequent copy number alterations including amplification of 3q26/28 and 11q13/22. A subgroup of oral cavity tumours with favourable clinical outcomes displayed infrequent copy number alterations in conjunction with activating mutations of HRAS or PIK3CA, coupled with inactivating mutations of CASP8, NOTCH1 and TP53. Other distinct subgroups contained loss-of-function alterations of the chromatin modifier NSD1, WNT pathway genes AJUBA and FAT1, and activation of oxidative stress factor NFE2L2, mainly in laryngeal tumours. Therapeutic candidate alterations were identified in most HNSCCs.

HNSCCs affect $\sim 600,000$ patients per year worldwide ${ }^{1}$. They are characterized by phenotypic, aetiological, biological and clinical heterogeneity. Smoking is implicated in the rise of HNSCC in developing countries, and the role of human papillomavirus (HPV) is emerging as an important factor in the rise of oropharyngeal tumours affecting non-smokers in developed countries ${ }^{2}$. Despite surgery, radiation and chemotherapy, approximately half of all patients will die of the disease. Risk stratification for HNSCC is by anatomic site, stage and histological characteristics of the tumour. Except for HPV status, numerous molecular and clinical risk factors that have been investigated have limited clinical utility.

Published genome-wide profiling studies of $\mathrm{HNSCC}^{3,4}$ are limited to single platforms. To generate an integrated genomic annotation of molecular alterations in HNSCC, The Cancer Genome Atlas (TCGA) has undertaken a comprehensive multi-platform characterization of 500 tumours with the a priori hypothesis of detecting somatic variants present in at least $5 \%$ of samples. Here, we report the results for analyses from the first 279 patients with complete data.

\section{Samples and clinical data}

The cohort consists primarily of tumours from the oral cavity $(n=172$ out of $279,62 \%)$, oropharynx ( $n=33$ out of $279,12 \%)$, and laryngeal sites ( $n=72$ out of $279,26 \%$ ) (Supplementary Information section 1, Supplementary Table 1.1 and Supplementary Data 1.1). Most patients were male ( $n=203$ out of $279,73 \%$ ) and heavy smokers (mean pack years $=51$ ). Samples were classified as HPV-positive using an empiric definition of $>1,000$ mapped RNA sequencing (RNA-Seq) reads, primarily aligning to viral genes E6 and E7 (Supplementary Information section 1.2 and Supplementary Fig. 1.1). The HPV status by mapping of RNA-Seq reads was concordant with the genomic, sequencing and molecular data, and indicated that 36 tumours were HPV $(+)$ and 243 were $\mathrm{HPV}(-)$ (Supplementary Information section 1.2, Supplementary Fig. 1.1 and Supplementary Data 1.2). Of 33 oropharyngeal tumours, $64 \%$ were positive for HPV, compared to $6 \%$ of 246 non-oropharyngeal tumours. Molecular HPV signatures were identified using microRNA
(miRNA), DNA methylation, gene expression and somatic nucleotide substitutions (Supplementary Information section 1.2 and Supplementary Figs 1.1-1.3). HPV (+) tumours exhibited infrequent mutations in TP53 or genetic alterations in CDKN2A. We evaluated outcome by site, stage, HPV status, molecular subtypes and putative biomarkers (Supplementary Information section 1.3 and Supplementary Figs 1.4 and 1.5). $\mathrm{HPV}(+)$ and interestingly patients with $\mathrm{HPV}(-)$, TP53 wild-type tumours demonstrated favourable outcomes compared to TP53 mutants and 11q13/CCND1-amplified tumours.

\section{DNA and RNA structural alterations}

Most tumours demonstrated copy number alterations (CNAs) including losses of $3 p$ and $8 p$, and gains of $3 q, 5 p$ and $8 q$ chromosomal regions (Fig. 1a, Supplementary Fig. 2.1 and Supplementary Information section 2) resembling lung squamous cell carcinomas (LUSCs) ${ }^{5}$ (Fig. 1a and Supplementary Figs 2.1 and 2.2). HNSCC genomes showed high instability with a mean of 141 CNAs (amplifications or deletions) from microarray data and 62 structural aberrations (chromosomal fusions) per tumour by 'high coverage' whole-genome sequencing $(n=29)$ (Supplementary Information section 2.2). We observed 39 regions of recurrent copy number loss and 23 regions of recurrent copy number gain ( $q$ $<0.1$, Supplementary Data 2.1 and 2.2). Both HPV $(+)$ and $(-)$ tumours contained recurrent focal amplifications for $3 q 26 / 28$, a region involving squamous lineage transcription factors TP63 and SOX2 and the oncogene PIK3CA (Fig. 1b and Supplementary Fig. 2.3).

$\mathrm{HPV}(+)$ tumours were distinguished by novel recurrent deletions ( $n=5$ out of $36,14 \%)$ and truncating mutations $(n=3$ out of $36,8 \%)$ of TNF receptor-associated factor 3 (TRAF3) (Supplementary Figs 2.3 and 2.4, and Supplementary Data 2.1). TRAF3 is implicated in innate and acquired anti-viral responses ${ }^{6}$ including Epstein-Barr, HPV and human immunodeficiency virus (HIV $)^{7-9}$, while loss promotes aberrant NF- $\kappa \mathrm{B}$ signalling ${ }^{10}$. Although TRAF3 inactivation has been reported in haematological malignancies and nasopharyngeal carcinoma ${ }^{11,12}$, to our knowledge this is the first evidence linking TRAF3 to HPV-associated 

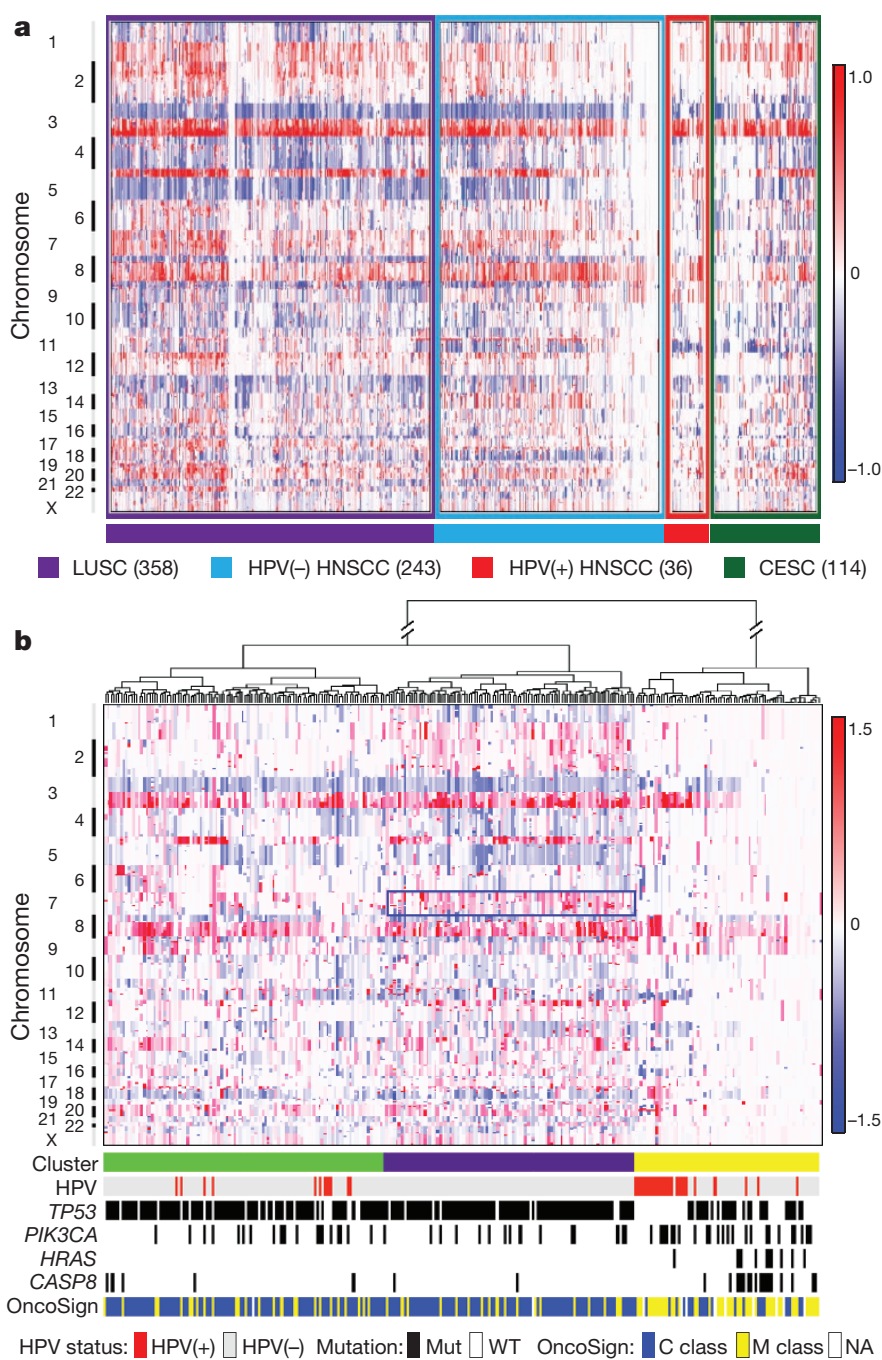

Figure $1 \mid$ DNA copy number alterations. a, Copy number alterations by anatomic site and HPV status for squamous cancers. Lung squamous cell carcinoma (LUSC, $n=358$ ) and cervical squamous cell carcinoma (CESC, $n=114)$. $\mathbf{b}$, Unsupervised analysis of copy number alteration of HNSCC $(n=279)$ with associated characteristics. The rectangle indicates chromosome 7 amplifications in the purple cluster. NA, not available.

carcinomas. $\mathrm{HPV}(+)$ tumours were also notable for focal amplification of $E 2 F 1$ and an intact 9p21.3 region containing the CDKN2A gene commonly deleted in $\mathrm{HPV}(-)$ tumours.

$\mathrm{HPV}(-)$ tumours featured novel co-amplifications of 11q13 (CCND1, FADD and CTTN) and 11q22 (BIRC2 and YAP1), which also contain genes implicated in cell death/NF- $\kappa \mathrm{B}$ and Hippo pathways. $\mathrm{HPV}(-)$ tumours featured novel focal deletions in the nuclear set domain gene (NSD1) and tumour suppressor genes (for example, FAT1, NOTCH1, $S M A D 4$ and CDKN2A; Supplementary Fig. 2.3). Recurrent focal amplifications in receptor tyrosine kinases (for example, EGFR, ERBB2 and FGFR1) also predominated in HPV(-) tumours. Notably, unsupervised clustering analysis of CNAs identified a mutually exclusive subset of predominantly oral cavity tumours with reduced CNAs, a pattern recently described in cancer as ' $M$ ' class (tumours driven by mutation rather than $\mathrm{CNA})^{13}$ (Fig. 1b). This subset in particular contained a new three-gene pattern of activating mutations in HRAS, frequently with inactivating CASP8 mutations, and wild-type TP53. We confirmed a previously reported favourable clinical outcome in tumours with few $\mathrm{CNAs}^{14}$. The three-gene constellation of wild-type TP53 with mutant HRAS and CASP8 suggested an alternative tumorigenesis pathway involving RAS and/or alterations in cell death/NF- $\kappa B^{15}$. Unsupervised analysis also suggested that clustering was a function of chromosome 7 amplification (including the EGFR locus) in a manner that largely excluded HPV $(+)$ tumours.

To detect additional structural alterations, we interrogated wholegenome and RNA-Seq data (Supplementary Information section 3, Supplementary Data 3.1 and Supplementary Fig. 3.1). Known fusion oncogenes reported in solid tumours including those involving the $A L K$, $R O S$ or RET genes were not observed in HNSCC. Previously reported FGFR3-TACC3 fusions were present in two HPV(+) tumours (Supplementary Fig. 3.2). Only 1 out of 279 patients showed evidence of the type III isoform of EGFR (vIII), previously described in $\mathrm{HNSCC}^{16}$ (Supplementary Fig. 3.3). Although our investigation did not identify additional novel oncogenic fusions, several tumours demonstrated exon 1 of EGFR or FGFR3 fused to non-recurrent partners, suggesting potential promoter swaps for the partner genes (Supplementary Data 3.1). A low prevalence of an alternative MET transcript with skipped exon 14 was identified in two HPV $(-)$ tumours (Supplementary Fig. 3.4); this finding was reported to be an activating event in non-small cell lung cancer ${ }^{17}$. Structural alterations (homozygous deletions, intra- and interchromosomal fusions) were more commonly associated with loss of function in tumour suppressor genes, most prominently CDKN2A (Supplementary Figs 3.5 and 3.6), followed by TP53, RB1, NOTCH1 and FAT1 (Supplementary Figs 3.7-3.9), than with protein-coding fusion events. RNA-Seq data (Supplementary Data 3.3) demonstrated evidence of alternative splicing in genes not previously described in HNSCC including kallikrein 12 (KLK12) (Supplementary Fig. 3.11), as well as genes such as TP63 with known importance in HNSCC (Supplementary Fig. 3.12).

By DNA analysis, most HPV $(+)$ tumours demonstrated clear evidence of host genome integration, usually in a single genomic location per sample and almost always in association with amplifications of the host genome (Supplementary Fig. 3.10 and Supplementary Data 3.2). Interrogation of RNA transcripts confirmed transcription across the viral-human integration locus. However, none of the genes involved were recurrent, suggesting no single driver mechanism related to HPV integration. Similarly, none of the integration sites involved the MYC gene as reported in $\operatorname{HPV}(+)$ cell lines ${ }^{18}$.

\section{Somatic mutations}

Whole-exome sequencing identified somatically mutated genes, many located in regions of CNAs and annotated in the COSMIC database ${ }^{19}$ (Fig. 2). The mean sequencing coverage across targeted bases was $95 \times$, with $82 \%$ of target bases above $30 \times$ coverage. In 279 samples, 12,159 synonymous somatic variants, 37,061 non-synonymous somatic variants, and 2,579 germline single base substitutions from the single nucleotide polymorphism database $(\mathrm{dbSNP})^{20}$ were detected (Supplementary Information section 4). Targeted re-sequencing of 394 unique regions (Supplementary Fig. 4.1) validated 99\% of mutations. Interrogation of RNA for expression of the mutated alleles confirmed the variant in $86 \%$ of cases (Supplementary Information section 3.2 and Supplementary Fig. 3.1). In contrast to previous reports, the mutation rates did not differ by HPV status, although transversions at $\mathrm{CpG}$ sites were more frequent in $\mathrm{HPV}(-)$ tumours and a predominance of TpC mutations were noted in HPV $(+)$ cases $^{3}$ (Supplementary Fig. 1.1). Mutations were statistically enriched in 11 genes (Fig. 2). Among inactivating mutations (premature termination of the protein by nonsense, frameshift or splice-site mutations), four genes segregated exclusively or predominantly in $\mathrm{HPV}(-)$ tumours. Two were associated with cell cycle and survival $(C D K N 2 A(P<0.01)$ and TP53 $(P<0.01))$ and two were linked to $\mathrm{Wnt} / \beta$-catenin signalling $(F A T 1(P<0.01)$ and $A J U B A$ $(P=0.14))^{21,22}$. We observed TP53 mutation among HPV $(-)$ samples at higher rates $(86 \%)$ than have been previously reported ${ }^{19}$, while only 1 out of $36 \mathrm{HPV}(+)$ cases had a non-synonymous TP53 mutation. Previously unreported somatic mutations and deletions of AJUBA were primarily $5^{\prime}$ inactivating events and clustered missense mutations in the functional LIM domain (Supplementary Fig. 4.2). AJUBA is a 


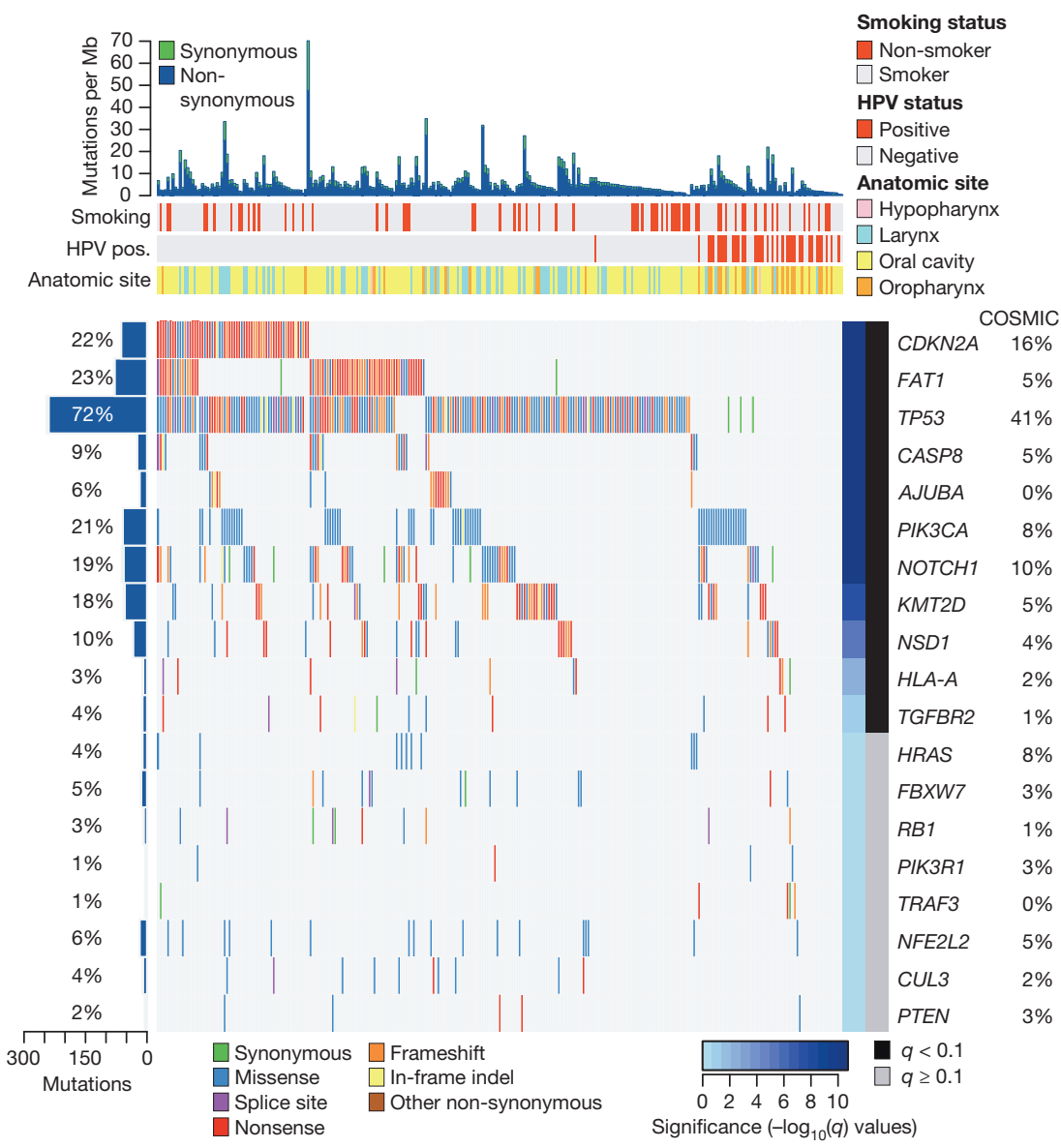

Figure $2 \mid$ Significantly mutated genes in HNSCC. Genes (rows) with significantly mutated genes (identified using the MutSigCV algorithim; $q<0.1$ ) ordered by $q$ value; additional genes with trends towards significance are also shown. Samples (columns, $n=279$ ) are arranged to emphasize mutual exclusivity among mutations. Left, mutation percentage in TCGA. Right, mutation percentage in COSMIC ('upper aerodigestive tract' tissue). Top, overall number of mutations per megabase. Colour coding indicates mutation type. centrosomal protein that regulates cell division, vertebrate ciliogenesis and left-right axis determination ${ }^{23}$. Additionally, AJUBA is subject to EGFR-RAS-MAPK-dependent phosphorylation and implicated in Hippo growth and regeneration pathways conserved from Drosophila to mammals ${ }^{24,25}$, in ataxia-telangiectasia mutated (ATM) and ATM and Rad-3related (ATR)-mediated DNA damage response ${ }^{26}$, and tumour invasion and migration ${ }^{27}$.

A frequently mutated novel gene, the nuclear receptor binding SET domain protein 1 (NSD1), was identified in 33 HNSCCs. Alterations included inactivating mutations $(n=29)$ and focal homozygous deletions $(n=4)$. NSD1 is a histone 3 Lys 36 (H3K36) methyltransferase, similar to SETD2, which is frequently mutated in the clear cell variant of renal cell carcinoma, and associated with DNA hypomethylation ${ }^{28}$. Germline carriers of inactivating mutations in NSD1 are associated with craniofacial abnormalities (Sotos syndrome), and malignancies including squamous carcinoma, implicating NSD1 as a tumour suppressor gene $^{29}$. Interestingly, NSD1 functions as an oncogene when fused to nucleoporin-98 (NUP98) $\mathrm{t}(5 ; 11)(\mathrm{q} 35 ; \mathrm{p} 15.5)$ in haematological cancers with increased $\mathrm{H} 3 \mathrm{~K} 36$ trimethylation levels at $\mathrm{HOX}$ A genes and accompanying transcriptional activation ${ }^{30}$. Translocations involving other dedicated H3K36 methyltransferase genes including WHSC1 (also known as MMSET and NSD2) are reported in 20\% of multiple myelomas. By contrast, NSD1 loss has been associated with sporadic non-melanoma skin cancers ${ }^{31}$. Significant inactivating mutations were found in genes linked to squamous differentiation including in NOTCH1 (19\%), and other non-significant family members (NOTCH2 9\%, and NOTCH3 $5 \%, q>0.1$, non-significant), and the TP63 target gene ZNF750 (4\%, $q>0.1$, non-significant), which falls in a significantly deleted peak at 17q25.3. The analysis identified additional mutations including TRAF3, $R B 1$ and NFE2L2, among others with $q$ values $<1$ (non-significant). The frequently mutated apoptosis gene CASP8 displayed clustered missense and other inactivating mutations in the first death effector, intron and caspase peptidase domains. Statistically significant mutations in KMT2D (also known as MLL2) and HLA-A could contribute to defective immunosurveillance. Of known oncogenes, only PIK3CA achieved statistical significance $(q<0.01)$. Approximately one-quarter of the mutated PIK3CA cases displayed concurrent amplification, with an additional $20 \%$ of tumours containing focal amplification without evidence of mutation. Seventy-three per cent of PIK3CA mutations localized to Glu542Lys, Glu545Lys and His1047Arg/Leu hotspots that promote activation, with the remaining mutations of uncertain function. Recurrent activating mutations of HRAS in the GTPase domain in residues 11-13 approached statistical significance $(q=0.2)$.

We extended our unsupervised genome-wide analysis of significantly mutated genes as well as genes reported in COSMIC to a subgroup analysis by anatomic sites, tumour versus normal status, HPV status and four previously validated gene expression subtypes ${ }^{32,33}$ (Supplementary Information section 5, Supplementary Figs 5.1-5.4 and Supplementary Data 4.1 and 5.1-5.4). Additional mutations included TRAF3, RB1 and NFE2L2, among others with $q$ values $<1$, and we observed statistical evidence for mutations of HRAS ( $q=0$ in COSMIC subset) and other genes. Sporadic inactivating mutations and deletions of TGFBR2 were identified primarily in oral cavity tumours, consistent with its role in promoting squamous tumorigenesis in mouse models ${ }^{34}$. Investigating COSMIC database mutations focused attention on the significant deletion peak at $4 \mathrm{q} 31.3$ containing the gene $F B X W 7$, a ubiquitin ligase targeting cyclin $\mathrm{E}$ and $\mathrm{NOTCH}$ genes, in which we identified mutations that included recurrent Arg505Gly/Leu substitutions $(n=14)$. Genes with at least one identical mutation previously reported in COSMIC include SCN9A, CHEK2, PTCH1 and PIK3R1. We further focused on somatic alterations and protein expression that represent plausible therapeutic targets (Fig. 3, Supplementary Information sections 6 and 7, Supplementary Figs 6.1 and 6.2 and Supplementary Data 6.1 and 6.2). 


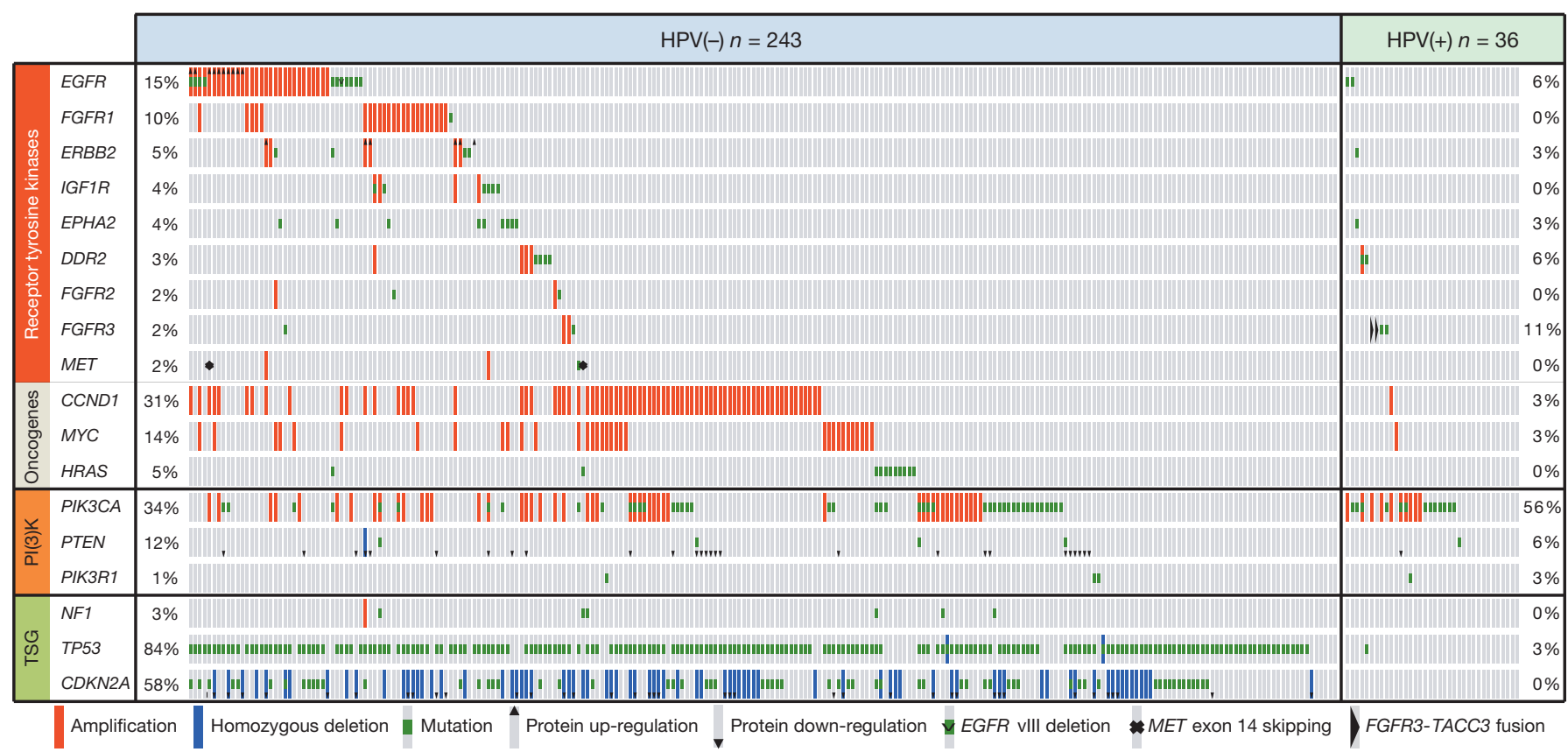

Figure $3 \mid$ Candidate therapeutic targets and driver oncogenic events. Alteration events for key genes are displayed by sample ( $n=279)$. TSG, tumour suppressor gene.

\section{Integrated genome analysis and pathways}

Correlative genetic alteration analysis identified numerous pairwise significant findings (Supplementary Information section 7 and Supplementary Fig. 7.1). In particular, co-amplification of 11q13 containing CCND1, FADD and CTTN and a narrow segment of 11q22 containing the genes with equal evidence for YAP1 and BIRC2 was further characterized (Supplementary Fig. 7.2). Chromosome 11q22 was focally but rarely amplified in the absence of co-amplification of 11q13. This novel finding suggests that the selection pressure for this co-amplification stems from the interaction of BIRC2 with FADD and the caspase cascade that inhibits cell death. Notably, the vast majority of tumours with the 11q13 amplification had large deletions in the telomeric region of 11q22, including other genes known to be important in cell death in cancer such as ATM and CASP1, 4, 5 and 12. Amplification of 11q13 was anti-correlated with CASP8 mutations, suggesting an alternative function of CASP8 and FADD in cell death/NF- $\mathrm{\kappa B}$ activation ${ }^{35}$.
We investigated whether clinical factors, single gene alterations and statistically significant pairwise gene correlations (Supplementary Fig. 7.1 and Supplementary Data 7.1) might segregate previously defined molecular subtypes and/or anatomic sub-sites (Supplementary Data 5.1-5.4). We confirmed reported gene expression subtypes (atypical (24\%), mesenchymal (27\%), basal (31\%) and classical (18\%)), and assessed the subtypes for enrichment of somatic alterations ${ }^{32,33}$ (Supplementary Data 7.1). Notably, TP53 mutation, CDKN2A loss of function, chromosome $3 \mathrm{q}$ amplification, alteration of oxidative stress genes (KEAP1, NFE2L2 or CUL3), heavy smoking history (Supplementary Table 1.1) and larynx sub-site co-occurred in most classical subtype tumours (Fig. 4a and Supplementary Information section 7.2), similar to LUSC $^{5}$ (Supplementary Figs 5.1 and 5.2). Collectively, these findings suggest that the NFE2L2 oxidative stress pathway is a tobacco-related signature across anatomic tumour sites. By contrast, the basal subtype demonstrated inactivation of $\mathrm{NOTCH} 1$ with intact oxidative stress a

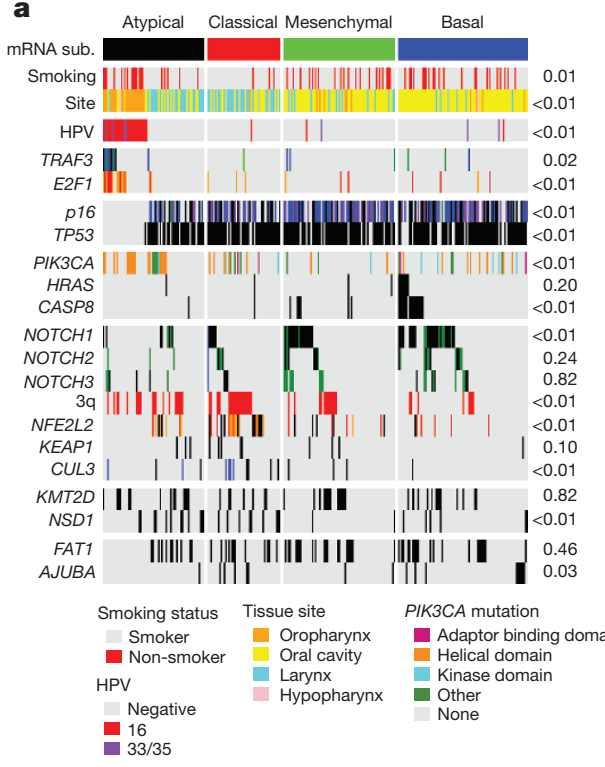

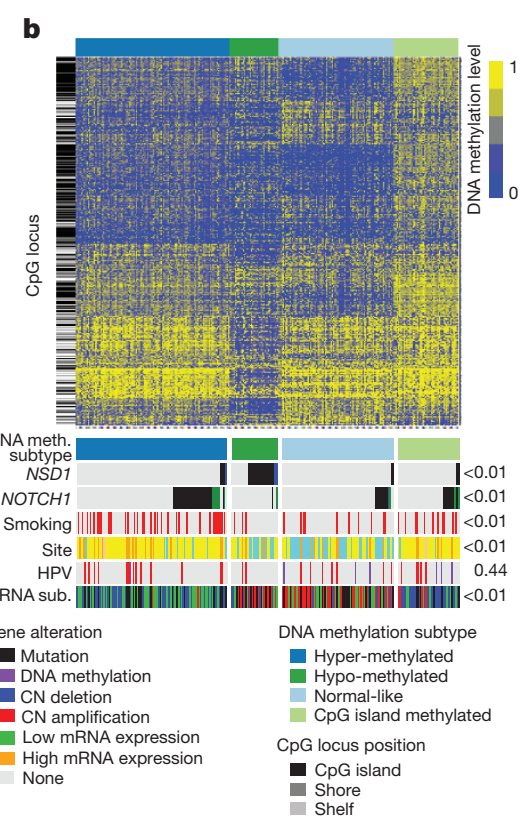

Figure $4 \mid$ Integrated analysis of genomic alterations. a, b, Samples $(n=279)$ are displayed in columns and grouped by gene expression (a) or methylation (b) subtype (sub.). Unadjusted two-sided Fisher's exact test $P$ values assess the association of each genomic alteration. Methylation probe location of CpG islands, shores and shelves are shown on the left of $\mathbf{b}$. Annotation shows HPV status and subtype (16, 33 and 35$)$. CN, copy number. 


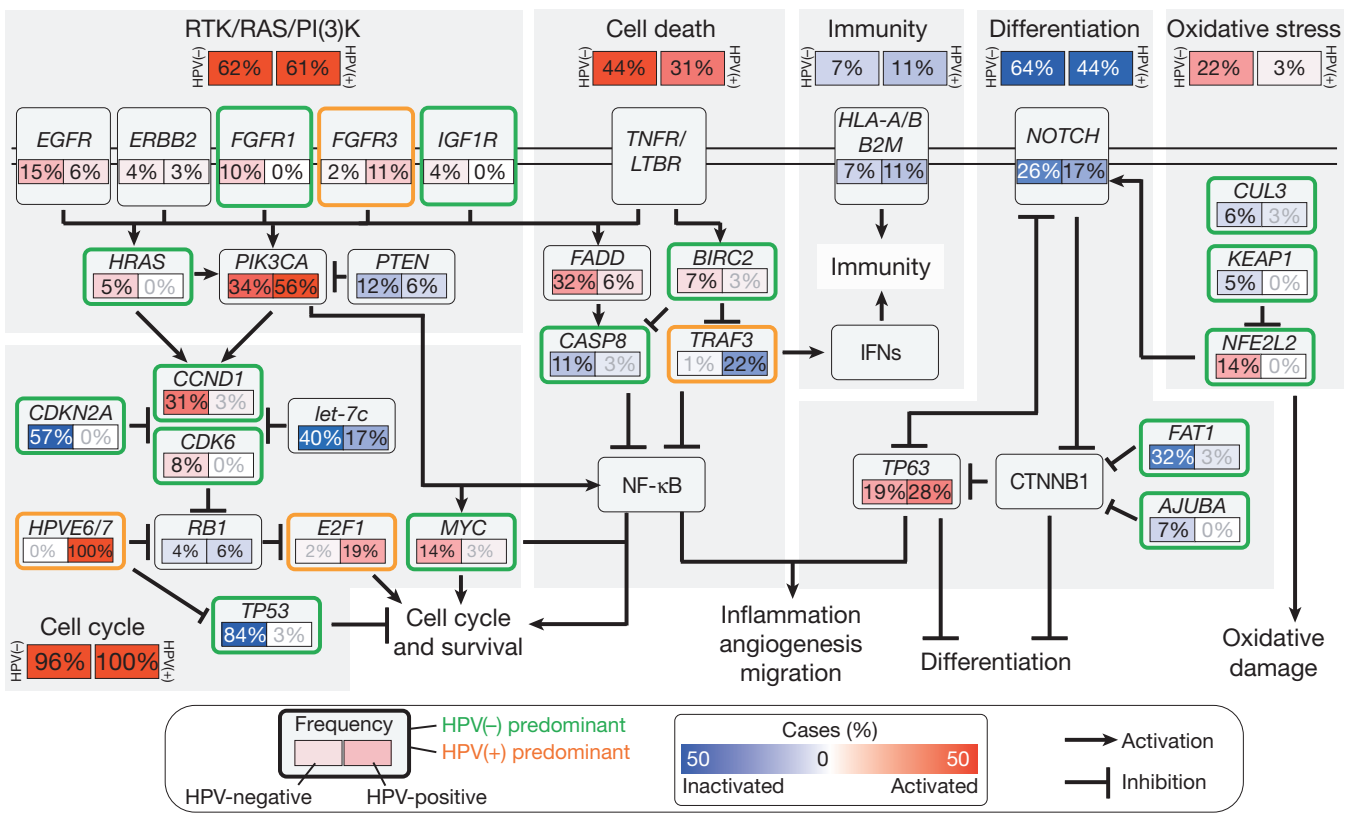

Figure 5 | Deregulation of signalling pathways and transcription factors. Key affected pathways, components and inferred functions, are summarized in the main text and Supplementary Information section 7 for $n=279$ samples. The frequency (\%) of genetic alterations for $\mathrm{HPV}(-)$ and $\mathrm{HPV}(+)$ tumours are shown separately within sub-panels and highlighted. Also see Supplementary Fig. 7.15. Pathway alterations include homozygous deletions, focal amplifications and somatic mutations. Activated and inactivated pathways/genes, and activating or inhibitory symbols are based on predicted effects of genome alterations and/or pathway functions. signalling and fewer alterations of chromosome 3q. Analysis of the 3q locus highlighted a marked relative decrease of SOX2 expression in basal tumours relative to all other HNSCC and tumour adjacent normal samples (Supplementary Fig. 5.4), supporting the interaction of transcription factors SOX2, TP63, NFE2L2 and NOTCH1 as driving differences between expression subtypes. Additionally, the basal subtype included most tumours with the HRAS-CASP8 co-mutation and most co-amplified 11q13/q22 tumours. These findings along with HRAS mutations implicate disrupted cell death as a major alteration in this subtype $^{36}$ (Supplementary Fig. 7.2). The atypical subtype was characterized by a lack of chromosome 7 amplifications (Supplementary Fig. 5.3), enrichment of $\mathrm{HPV}(+)$ tumours with activating mutations in exon 9 that contains the PIK3CA helical domain. By contrast, the mesenchymal subtype showed high levels of alteration in innate immunity genes, in particular high expression of natural killer cell marker CD56 and a low frequency of HLA class I mutations (Supplementary Fig. 7.3). Among the significantly mutated genes, TP53 $(P<0.001), C A S P 8(P=$ $0.01)$, NSD1 $(P=0.01)$ and $C D K N 2 A(0.06)$ were the most differentially mutated across anatomic sites (Supplementary Data 4.1). Most CASP8 mutations (22 out of $24,92 \%)$ were in oral cavity tumours, whereas TP53, NSD1 and CDKN2A demonstrated decreased mutation rates in oropharyngeal tumours relative to other sites.

Unsupervised analysis of gene expression by HPV status and of reverse-phase protein arrays (Supplementary Information section 6), DNA methylation (Supplementary Information section 8), and miRNA platforms (Supplementary Information section 9, Supplementary Table 9.1, Supplementary Figs 7.4-7.9 and Supplementary Data 7.1) showed high correlation across platforms $(P<0.01$; Fig. 4 , Supplementary Information section 7.9 and Supplementary Data 7.2) and coordinated alterations of genes including the epithelial-mesenchymal transition signature $^{37}$ (Supplementary Figs 7.4, 7.7 and 7.8). However, within the broader cross-platform agreement, individual unsupervised clustering of miRNA, reverse-phase protein arrays and DNA methylation data provided insight into the association of molecular subtypes with single gene alterations. The most notable example was the detection of hypomethylation and loss-of-function mutations of NSD1, and wild-type NOTCH1 in atypical and classical gene expression subtypes (Fig. $4 \mathrm{~b}$ and Supplementary Table 7.1).

Supervised analyses detected genomic features (miRNA, gene expression and DNA methylation) associated with anatomic site (Supplementary Figs 5.5-5.8 and Supplementary Data 5.1-5.4). A supervised integrated analysis identified target genes that are inversely regulated by
miRNAs in HNSCC (Supplementary Information section 7.4). Among these miRNA-messenger RNA networks, let-7c-5p and miR-100-5p exhibited a correlation between low copy number and expression. Let-7c-5p and miR-100-5p were decreased in tumours compared to normal (Supplementary Fig. 7.10). For these miRNAs, deletion was highly associated with increased expression of target genes, including the cell cycle regulator $C D K 6$, transcription factor $E 2 F 1$ (ref. 38), mitosis regulator PLK1 (ref. 39), and transcription factor HMGA2 (ref. 40; Supplementary Figs 7.10, Supplementary Tables 7.2 and 7.3).

Integrative bioinformatics analysis identified a limited number of pathways targeted by frequent genome alterations (Fig. 5, Supplementary Information section 7, Supplementary Figs 7.11-7.15 and Supplementary Data 7.3). Among receptor tyrosine kinases, EGFR/ERBB2 or FGFR1/3 alterations are the most frequent. Among downstream targets of the receptor tyrosine kinase (RTK)/RAS/phosphatidylinositol3-OH kinase $(\mathrm{PI}(3) \mathrm{K})$ pathway, PIK3CA dominates with occasional HRAS and PTEN alterations. Further downstream, nearly every tumour has alteration of genes governing the cell cycle. The tumour suppressors TP53 and CDKN2A, oncogenes CCND1 and MYC, and the newly identified miRNA let-7c, are most often altered in $\operatorname{HPV}(-)$ tumours, whereas viral genes $E 6, E 7$ and $E 2 F 1$ predominate in $\mathrm{HPV}(+)$ cases. In addition, we report frequent alterations in genes involved in cell death, NF$\kappa \mathrm{B}$-mediated survival, or immunity pathways ${ }^{15,35}$. Co-amplification of $F A D D \pm B I R C 2$, or CASP \pm HRAS mutations define exclusive HPV $(-)$ subsets, whereas TRAF3 loss characterizes an HPV $(+)$ subset. These alterations along with PIK3CA and TP63 converge on NF- $\kappa \mathrm{B}$ transcription factors that promote cell survival, migration, inflammation and angiogenesis $^{41,42}$. Furthermore, TRAF3 and/or HLA loss are implicated in deregulation of innate antiviral and adaptive anti-tumour immunity ${ }^{43,44}$. Further alterations of NOTCH, TP63 and other genes in $\mathrm{HPV}(-)$ tumours (FAT1 and $A J U B A)$ recently linked functionally to $\beta$-catenin $(C T N N B 1)$ are also detected ${ }^{21,22,45}$. Finally, we highlight a previously underappreciated role for a key transcription factor regulator of oxidative stress, NFE2L2, and its protein complex partners CUL3 and KEAP1 in $\mathrm{HPV}(-) \mathrm{HNSCCs}$.

\section{Conclusion}

The TCGA study represents the most comprehensive integrative genomic analysis of HNSCC. Loss of TRAF3, activating mutations of $P I K 3 C A$, and amplification of $E 2 F 1$ in $\mathrm{HPV}(+)$ oropharyngeal cancers point to aberrant activation of NF- $\kappa \mathrm{B}$, other oncogenic pathways, and cell cycle, as critical in the pathogenesis and development of new 
targeted therapies for these tumours. In HPV(-) HNSCCs, mutually exclusive subsets containing amplicons on 11q with CCND1, FADD, $B I R C 2$ and $Y A P 1$, or concurrent mutations of CASP 8 with HRAS, also target cell cycle, death, NF- $\kappa \mathrm{B}$ and other oncogenic pathways. Recent studies predict that the inactivation of $A J U B A$, as well as FAT1 and NOTCH1, may converge to uncheck $\mathrm{Wnt} / \beta$-catenin signalling, implicated in deregulation of cell polarity and differentiation. The $3 \mathrm{q}$ amplicon found in both HPV $(+)$ and $(-)$ HNSCCs includes transcription factors TP63, SOX2 and signal molecule PIK3CA, which are also implicated in homeostasis of epithelial stem cells and differentiation. Among these, the biological function and agents targeting BIRCs, PI(3)K, Wnt/ $\beta$-catenin and NOTCH are under investigation. Collectively, these findings provide new insights into HNSCC and suggest that shared and unique alterations might be leveraged to accelerate progress in prevention and therapy across tumour types.

\section{Received 19 October 2013; accepted 1 December 2014.}

1. Ferlay, J. et al. Estimates of worldwide burden of cancer in 2008: GLOBOCAN 2008. Int. J. Cancer 127, 2893-2917 (2010).

2. Ang, K. K. et al. Human papillomavirus and survival of patients with oropharyngeal cancer. N. Engl. J. Med. 363, 24-35 (2010).

3. Stransky, N. et al. The mutational landscape of head and neck squamous cell carcinoma. Science 333, 1157-1160 (2011)

4. Agrawal, N. et al. Exome sequencing of head and neck squamous cell carcinoma reveals inactivating mutations in NOTCH1. Science 333, 1154-1157 (2011).

5. The Cancer Genome Atlas Research Network. Comprehensive genomic characterization of squamous cell lung cancers. Nature 489, 519-525 (2012).

6. Oganesyan, G. et al. Critical role of TRAF3 in the Toll-like receptor-dependent and -independent antiviral response. Nature 439, 208-211 (2006).

7. Karim, R. et al. Human papillomavirus (HPV) upregulates the cellular deubiquitinase UCHL1 to suppress the keratinocyte's innate immune response. PLOS Pathog. 9, e1003384 (2013).

8. Eliopoulos, A. G. et al. CD40-induced growth inhibition in epithelial cells is mimicked by Epstein-Barr Virus-encoded LMP1: involvement of TRAF3 as a common mediator. Oncogene 13, 2243-2254 (1996).

9. Imbeault, M. et al. Acquisition of host-derived CD4OL by HIV-1 in vivo and its functional consequences in the B-cell compartment. J. Virol. 85, 2189-2200 (2011).

10. Ni, C. Z. et al. Molecular basis for $C D 40$ signaling mediated by TRAF3. Proc. Natl Acad. Sci. USA 97, 10395-10399 (2000).

11. Chung, G. T. et al. Constitutive activation of distinct NF-kB signals in EBVassociated nasopharyngeal carcinoma. J. Pathol. 231, 311-322 (2013).

12. Annunziata, C. M. etal. Frequent engagement of the classical and alternative NF-кB pathways by diverse genetic abnormalities in multiple myeloma. Cancer Cell 12, 115-130 (2007).

13. Ciriello, G. et al. Emerging landscape of oncogenic signatures across human cancers. Nature Genet. 45, 1127-1133 (2013).

14. Smeets, S. J. et al. Genetic classification of oral and oropharyngeal carcinomas identifies subgroups with a different prognosis. Cellular Oncol. 31, 291-300 (2009).

15. Mayo, M. W. et al. Requirement of NF-kB activation to suppress p53-independent apoptosis induced by oncogenic Ras. Science 278, 1812-1815 (1997).

16. Sok, J. C. et al. Mutant epidermal growth factor receptor (EGFRvIII) contributes to head and neck cancer growth and resistance to EGFR targeting. Clin. Cancer Res. $12,5064-5073$ (2006)

17. Kong-Beltran, M. et al. Somatic mutations lead to an oncogenic deletion of met in lung cancer. Cancer Res. 66, 283-289 (2006).

18. Popescu, N. C., DiPaolo, J. A. \& Amsbaugh, S. C. Integration sites of human papillomavirus 18 DNA sequences on HeLa cell chromosomes. Cytogenet. Cell Genet. 44, 58-62 (1987).

19. Forbes, S. A. et al. The Catalogue of Somatic Mutations in Cancer (COSMIC). Curr. Protoc. Hum. Genet. Chapter 10, Unit 10.11 (2008).

20. Sherry, S. T. etal. dbSNP: the NCBI database of genetic variation. Nucleic Acids Res. 29, 308-311 (2001).

21. Morris, L. G. et al. Recurrent somatic mutation of FAT1 in multiple human cancers leads to aberrant Wnt activation. Nature Genet. 45, 253-261 (2013).

22. Haraguchi, K. et al. Ajuba negatively regulates the Wnt signaling pathway by promoting GSK-3 $\beta$-mediated phosphorylation of $\beta$-catenin. Oncogene $\mathbf{2 7}$, 274-284 (2008).

23. Nagai, Y. et al. The LIM protein Ajuba is required for ciliogenesis and left-right axis determination in medaka. Biochem. Biophys. Res. Commun. 396, 887-893 (2010).

24. Sun, G. \& Irvine, K. D. Ajuba family proteins link JNK to Hippo signaling. Sci. Signal. 6, ra81 (2013).

25. Reddy, B. V. \& Irvine, K. D. Regulation of Hippo signaling by EGFR-MAPK signaling through Ajuba family proteins. Dev. Cell 24, 459-471 (2013).

26. Kalan, S., Matveyenko, A. \& Loayza, D. LIM protein Ajuba participates in the repression of the ATR-mediated DNA damage response. Front. Genet. 4, 95 (2013).

27. Nola, S. et al. Ajuba is required for Rac activation and maintenance of $\mathrm{E}$-cadherin adhesion. J. Cell Biol. 195, 855-871 (2011).

28. The Cancer Genome Atlas Research Network. Comprehensive molecular characterization of clear cell renal cell carcinoma. Nature 499, 43-49 (2013).
29. Fickie, M. R. etal. Adults with Sotos syndrome: review of 21 adults with molecularly confirmed NSD1 alterations, including a detailed case report of the oldest person. Am. J. Med. Genet. A. 155, 2105-2111 (2011).

30. Wang, G. G., Cai, L., Pasillas, M. P. \& Kamps, M. P. NUP98-NSD1 links H3K36 methylation to Hox-A gene activation and leukaemogenesis. Nature Cell Biol. 9, 804-812 (2007).

31. Quintana, R. M. etal. A transposon-based analysis of gene mutations related to skin cancer development. J. Invest. Dermatol. 133, 239-248 (2013).

32. Chung, C. H. et al. Molecular classification of head and neck squamous cell carcinomas using patterns of gene expression. Cancer Cell 5, 489-500 (2004).

33. Walter, V. et al. Molecular subtypes in head and neck cancer exhibit distinct patterns of chromosomal gain and loss of canonical cancer genes. PLOS ONE $\mathbf{8}$, e56823 (2013).

34. Lu, S. L. et al. Loss of transforming growth factor- $\beta$ type II receptor promotes metastatic head-and-neck squamous cell carcinoma. Genes Dev. 20, 1331-1342 (2006).

35. Oberst, A. \& Green, D. R. It cuts both ways: reconciling the dual roles of caspase 8 in cell death and survival. Nature Rev. Mol. Cell Biol. 12, 757-763 (2011).

36. Park, S. J. et al. Opposite role of Ras in tumor necrosis factor- $\alpha$-induced cell cycle regulation: competition for Raf kinase. Biochem. Biophys. Res. Commun. 287, 1140-1147 (2001)

37. Byers, L. A. et al. An epithelial-mesenchymal transition gene signature predicts resistance to EGFR and PI3K inhibitors and identifies Axl as a therapeutic target for overcoming EGFR inhibitor resistance. Clin. Cancer Res. 19, 279-290 (2013).

38. Wong, J. V., Dong, P., Nevins, J. R., Mathey-Prevot, B. \& You, L. Network calisthenics: control of E2F dynamics in cell cycle entry. Cell Cycle 10, 3086-3094 (2011).

39. Sanhaji, M. et al. Polo-like kinase 1 inhibitors, mitotic stress and the tumor suppressor p53. Cell Cycle 12, 1340-1351 (2013).

40. Morishita, A. et al. HMGA2 is a driver of tumor metastasis. Cancer Res. $\mathbf{7 3}$, 4289-4299 (2013)

41. Bancroft, C. C. et al. Effects of pharmacologic antagonists of epidermal growth factor receptor, PI3K and MEK signal kinases on NF-KB and AP-1 activation and IL8 and VEGF expression in human head and neck squamous cell carcinoma lines. Int. J. Cancer 99, 538-548 (2002).

42. Yang, X. et al. $\Delta N p 63$ versatilely regulates a broad NF- $k B$ gene program and promotes squamous epithelial proliferation, migration, and inflammation. Cancer Res. 71, 3688-3700 (2011).

43. Keating, P. J. et al. Frequency of down-regulation of individual HLA-A and -B alleles in cervical carcinomas in relation to TAP-1 expression. Br. J. Cancer 72, 405-411 (1995).

44. Esteban, F. et al. Lack of MHC class I antigens and tumour aggressiveness of the squamous cell carcinoma of the larynx. Br. J. Cancer 62, 1047-1051 (1990).

45. Dotto, G. P. Crosstalk of Notch with p53 and p63 in cancer growth control. Nature Rev. Cancer 9, 587-595 (2009).

Supplementary Information is available in the online version of the paper.

Acknowledgements This study was supported by National Institutes of Health $(\mathrm{NIH})$ grants: P50CA097190, P50CA16672, U54 HG003273, U54 HG003067, U54 HG003079, U24 CA143799, U24 CA143835, U24 CA143840, U24 CA143843, U24 CA143845, U24 CA143848, U24 CA143858, U24 CA143866, U24 CA143867, U24 CA143882, U24 CA143883, U24 CA144025 and R01 CA 095419. Supported by The Bobby F. Garrett Fund for Head and Neck Cancer Research and NIDCD Intramural Projects ZIA-DC-000016, 73 and 74.

Author Contributions The TCGA research network contributed collectively to this study. Biospecimens were provided by the tissue source sites and processed by the biospecimen core resource. Data generation and analyses were performed by the genome sequencing centres, cancer genome characterization centres and genome data analysis centres. All data were released through the data coordinating centre. Project activities were coordinated by the National Cancer Institute and National Human Genome Research Institute project teams. We also acknowledge the following TCGA investigators who made substantial contributions to the project:

C.V.W. (manuscript coordinator); V.W. (data coordinator); P.S.H. and D.N.H. (analysis coordinators); Y.W. (data coordinating centre representative); R.A., J.B., T.E.C., J.J.L., M.Parfenov, A.D.T., V.W. and M.D.W. (clinical and HPV analysis); A.D.C. (DNA copy number analysis); J.Cho, A.H., M.Parfenov and M.D.W. (mutation calling); L.C. and L.Danilova (DNA methylation analysis); R.Bowlby, J.P.B., Z.C., H.Cheng, A.H., F.F.L., A.G.R., A.D.S. and C.V.W. (miRNA sequence analysis); L.A.B., L.Diao, P.K., W.L., S.N. and J.W. (reverse-phase protein arrays analysis); L.A.B., L.Diao, S.R.J., P.K.K., V.W., M.D.W. and N.Z. (mRNA sequence analysis); S.Benz, Z.C., R.L.F., S.N., C.R.P., N.Schultz, T.Y.S., C.V.W. and V.W. (pathway analysis); A.E.N., J.R.G. and D.N.H. (project chairs).

Author Information The primary and processed data used to generate the analyses can be downloaded by registered users from The Cancer Genome Atlas (https:// tcga-data.nci.nih.gov/tcga/tcgaDownload.jsp, https://cghub.ucsc.edu/ and https:// tcga-data.nci.nih.gov/docs/publications/hnsc_2014/). Reprints and permissions information is available at www.nature.com/reprints. The authors declare no competing financial interests. Readers are welcome to comment on the online version of the paper. Correspondence and requests for materials should be addressed to D.N.H. (hayes@med.unc.edu), J.R.G. (grandisjr@upmc.edu) or A.E.N. (anaggar@mdanderson.org).

This work is licensed under a Creative Commons Attributioncc)
third party material in this article are included in the article's Creative Commons licence, unless indicated otherwise in the credit line; if the material is not included under the Creative Commons licence, users will need to obtain permission from the licence holder 
to reproduce the material. To view a copy of this licence, visit http://creativecommons. org/licenses/by-nc-sa/3.0

The Cancer Genome Atlas Research Network (Participants are arranged by area of contribution and then by institution.)

Genome sequencing centre: Broad Institute Michael S. Lawrence ${ }^{1}$, Carrie Sougnez ${ }^{1}$, Lee Lichtenstein ${ }^{1}$, Kristian Cibulskis $^{1}$, Eric Lander ${ }^{1}$, Stacey B. Gabriel ${ }^{1}$, Gad Getz ${ }^{1,2}$

Genome characterization and data analysis centres: BC Cancer Agency Adrian $\mathrm{Ally}^{3}$, Miruna Balasundaram ${ }^{3}$, Inanc Birol ${ }^{3}$, Reanne Bowlby ${ }^{3}$, Denise Brooks ${ }^{3}$, Yaron S. N. Butterfield ${ }^{3}$, Rebecca Carlsen ${ }^{3}$, Dean Cheng ${ }^{3}$, Andy $\mathrm{Chu}^{3}$, Noreen Dhalla ${ }^{3}$, Ranabir Guin $^{3}$, Robert A. Holt ${ }^{3}$, Steven J. M. Jones ${ }^{3}$, Darlene Lee ${ }^{3}$, Haiyan I. Li ${ }^{3}$, Marco A. Marra ${ }^{3}$, Michael Mayo ${ }^{3}$, Richard A. Moore ${ }^{3}$, Andrew J. Mungall ${ }^{3}$, A. Gordon Robertson ${ }^{3}$, Jacqueline E. Schein ${ }^{3}$, Payal Sipahimalani ${ }^{3}$, Angela Tam ${ }^{3}$, Nina Thiessen ${ }^{3}$, Tina Wong ${ }^{3}$, Harvard Medical School/Brigham \& Women's Hospital/MD Anderson Cancer Center Alexei Protopopov ${ }^{4}$, Netty Santoso ${ }^{5}$, Semin Lee ${ }^{5}$, Michael Parfenov $v^{5}$, Jianhua Zhang ${ }^{4}$, Harshad S. Mahadeshwar ${ }^{4}$, Jiabin Tang ${ }^{4}$, Xiaojia Ren ${ }^{5}$, Sahil Seth ${ }^{4}$, Psalm Haseley ${ }^{5}$, Dong Zeng ${ }^{4}$, Lixing Yang ${ }^{5}$, Andrew W. Xu ${ }^{5}$, Xingzhi Song ${ }^{4}$, Angeliki Pantazi ${ }^{5}$, Christopher A. Bristow ${ }^{4}$, Angela Hadjipanayis ${ }^{5,6}$, Jonathan Seidman ${ }^{5}$, Lynda Chin ${ }^{1,4}$, Peter J. Park ${ }^{5,6,7}$, Raju Kucherlapati, ${ }^{5,6}$; The University of Texas MD Anderson Cancer Center Rehan Akbani ${ }^{4}$, Tod Casasent ${ }^{4}$, Wenbin Liu ${ }^{4}$, Yiling Lư ${ }^{4}$, Gordon Mills ${ }^{4}$, Thomas Motter ${ }^{4}$, John Weinstein ${ }^{4}$, Lixia Diao ${ }^{4}$, Jing Wang ${ }^{4}$, You Hong Fan ${ }^{4}$; University of Kentucky Jinze Liu ${ }^{8}$, Kai Wang ${ }^{8}$; University of North Carolina at Chapel Hill J. Todd Auman $^{9}$, Saianand Balu' ${ }^{9}$, Thomas Bodenheimer ${ }^{9}$, Elizabeth Buda ${ }^{9}$, D. Neil Hayes ${ }^{9}$, Katherine A. Hoadley ${ }^{9}$, Alan P. Hoyle ${ }^{9}$, Stuart R. Jefferys ${ }^{9}$, Corbin D. Jones ${ }^{9}$, Patrick K. Kimes $^{9}$, Yufeng Liu 9 , J. S. Marron ${ }^{9}$, Shaowu Meng ${ }^{9}$, Piotr A. Mieczkowski ${ }^{9}$, Lisle E. Mose ${ }^{9}$, Joel S. Parker ${ }^{9}$, Charles M. Perou ${ }^{9}$, Jan F. Prins ${ }^{9}$, Jeffrey Roach ${ }^{9}$, Yan Shi ${ }^{9}$, Janae V. Simons ${ }^{9}$, Darshan Singh ${ }^{9}$, Matthew G. Soloway ${ }^{9}$, Donghui Tan ${ }^{9}$, Umadevi Veluvolu ${ }^{9}$, Vonn Walter ${ }^{9}$, Scot Waring ${ }^{9}$, Matthew D. Wilkerson ${ }^{9}$, Junyuan W $\mathrm{Wu}^{9}$, Ni Zhao ${ }^{9}$; Broad Institute Andrew D. Cherniack ${ }^{1}$, Peter S. Hammerman ${ }^{1,10}$, Aaron D. Tward ${ }^{1,5}$, Chandra Sekhar Pedamallu, ${ }^{1,10}$, Gordon Saksena ${ }^{1}$, Joonil Jung ${ }^{1}$, Akinyemi I. Ojesina ${ }^{1,1^{\prime}}$, Scott L. Carter $^{1}$, Travis I. Zack ${ }^{1,10,11}$, Steven E. Schumacher ${ }^{1,10}$, Rameen Beroukhim ${ }^{1,5,10}$, Samuel S. Freeman ${ }^{1}$, Matthew Meyerson ${ }^{1,5,10}$, Juok Cho ${ }^{1}$, Lynda Chin ${ }^{1,4}$, Gad Getz ${ }^{1,2}$ Michael S. Noble ${ }^{1}$, Daniel DiCara ${ }^{1}$, Hailei Zhang ${ }^{1}$, David I. Heiman ${ }^{1}$, Nils Gehlenborg ${ }^{1,5}$ Doug Voet ${ }^{1}$, Pei Lin ${ }^{1}$, Scott Frazer ${ }^{1}$, Petar Stojanov ${ }^{1,10}$, Yingchun Liu ${ }^{1}$, Lihua Zou ${ }^{1}$ Jaegil Kim ${ }^{1}$, Carrie Sougnez ${ }^{1}$, Stacey B. Gabriel ${ }^{1}$, Michael S. Lawrence ${ }^{1}$; Baylor College of Medicine Donna Muzny ${ }^{12}$, HarshaVardhan Doddapaneni ${ }^{12}$, Christie Kovar ${ }^{12}$, Jeff Reid ${ }^{12}$, Donna Morton ${ }^{12}$, Yi Han ${ }^{12}$, Walker Hale ${ }^{12}$, Hsu Chao ${ }^{12}$, Kyle Chang ${ }^{12}$ Jennifer A. Drummond ${ }^{12}$, Richard A. Gibbs ${ }^{12}$, Nipun Kakkar ${ }^{12}$, David Wheeler ${ }^{12}$, Liu $\mathrm{X}^{12}$; Memorial Sloan-Kettering Cancer Center Giovanni Ciriello ${ }^{13}$, Marc Ladanyi ${ }^{13}$, William Lee $\mathrm{L}^{13}$, Ricardo Ramirez ${ }^{13}$, Chris Sander ${ }^{13}$, Ronglai Shen ${ }^{13}$, Rileen Sinha ${ }^{13}$, Nils Weinhold ${ }^{13}$, Barry S. Taylor ${ }^{13}$, B. Arman Aksoy ${ }^{13}$, Gideon Dresdner ${ }^{13}$, Jianjiong Gao ${ }^{13}$, Benjamin Gross ${ }^{13}$, Anders Jacobsen ${ }^{13}$, Boris Reva ${ }^{13}$, Nikolaus Schultz ${ }^{13}$, S. Onur Sumer ${ }^{13}$, Yichao Sun ${ }^{13}$, Timothy A. Chan ${ }^{13}$, Luc G. Morris ${ }^{13}$; University of California Santa Cruz/Buck Institute Joshua Stuart ${ }^{14}$, Stephen Benz ${ }^{14}$, Sam Ng ${ }^{14}$, Christopher Benz ${ }^{14}$, Christina Yau ${ }^{14}$; Johns Hopkins University/Sidney Kimmel Comprehensive Cancer Center Stephen B. Baylin ${ }^{15}$, Leslie Cope ${ }^{15}$, Ludmila Danilova ${ }^{15}$, James G. Herman ${ }^{15}$; University of Southern California Moiz Bootwalla ${ }^{16}$, Dennis T. Maglinte ${ }^{16}$ Peter W. Laird ${ }^{16}$, Timothy Triche Jr ${ }^{16}$, Daniel J. Weisenberger ${ }^{16}$, David J. Van Den Berg ${ }^{16}$

Disease working group: Nishant Agrawal ${ }^{17}$, Justin Bishop ${ }^{17}$, Paul C. Boutros ${ }^{18}$, Jeff $P$ Bruce $^{19}$, Lauren Averett Byers ${ }^{4}$, Joseph Califano ${ }^{17}$, Thomas E. Carey ${ }^{20}$, Zhong Chen ${ }^{21}$, Hui Cheng ${ }^{21}$, Simion I. Chiosea ${ }^{22}$, Ezra Cohen ${ }^{23}$, Brenda Diergaarde ${ }^{22}$, Ann Marie Egloff ${ }^{22}$, Adel K. El-Naggar ${ }^{4}$, Robert L. Ferris ${ }^{22}$, Mitchell J. Frederick' ${ }^{4}$, Jennifer R. Grandis $^{22}$, Yan Guo ${ }^{24}$, Robert I. Haddad ${ }^{10}$, Peter S. Hammerman ${ }^{1,10}$,'Thomas Harris ${ }^{25}$ D. Neil Hayes ${ }^{9}$, Angela B. Y. Hui ${ }^{19}$, J. Jack Lee ${ }^{4}$, Scott M. Lippman ${ }^{26}$, Fei-Fei Liu' ${ }^{27}$, Jonathan B. McHugh ${ }^{20}$, Jeff Myers ${ }^{4}$, Patrick Kwok Shing Ng${ }^{4}$, Bayardo

Perez-Ordonez ${ }^{28}$, Curtis R. Pickering ${ }^{4}$, Michael Prystowsky ${ }^{25}$, Marjorie Romkes ${ }^{22}$, Anthony D. Saleh ${ }^{21}$, Maureen A. Sartor ${ }^{20}$, Raja Seethala ${ }^{22}$, Tanguy Y. Seiwert ${ }^{23}$, Han $\mathrm{Si}^{21}$, Aaron D. Tward ${ }^{1,5}$, Carter Van Waes ${ }^{21}$, Daryl M. Waggott ${ }^{18}$, Maciej Wiznerowicz ${ }^{29}$ Wendell G. Yarbrough ${ }^{30}$, Jiexin Zhang ${ }^{4}$, Zhixiang Zuo ${ }^{23}$

Biospecimen core resource: International Genomics Consortium Ken Burnett ${ }^{31}$ Daniel Crain ${ }^{31}$, Johanna Gardner ${ }^{31}$, Kevin Lau ${ }^{31}$, David Mallery ${ }^{31}$, Scott Morris ${ }^{31}$, Joseph Paulauskis ${ }^{31}$, Robert Penny ${ }^{31}$, Candace Shelton ${ }^{31}$, Troy Shelton ${ }^{31}$, Mark Sherman ${ }^{31}$, Peggy Yena ${ }^{31}$; Nationwide Children's Hospital Aaron D. Black ${ }^{32}$, Jay Bowen $^{32}$, Jessica Frick ${ }^{32}$, Julie M. Gastier-Foster ${ }^{32}$, Hollie A. Harper ${ }^{32}$, Kristen Leraas ${ }^{32}$ Tara M. Lichtenberg ${ }^{32}$, Nilsa C. Ramirez ${ }^{32}$, Lisa Wise ${ }^{32}$, Erik Zmuda ${ }^{32}$

Data coordinating centre: Julien Baboud ${ }^{33}$, Mark A. Jensen ${ }^{33}$, Ari B. Kahn ${ }^{33}$, Todd D. Pih $^{33}$, David A. Pot ${ }^{33}$, Deepak Srinivasan ${ }^{33}$, Jessica S. Walton ${ }^{33}$, Yunhu Wan ${ }^{33}$
Project office: Robert A. Burton ${ }^{34}$, Tanja Davidsen ${ }^{35}$, John A. Demchok ${ }^{35}$, Greg Eley ${ }^{36}$ Martin L. Ferguson ${ }^{35}$, Kenna R. Mills Shaw ${ }^{35}$, Bradley A. Ozenberger ${ }^{37}$, Margi Sheth ${ }^{35}$, Heidi J. Sofia ${ }^{37}$, Roy Tarnuzzer ${ }^{35}$, Zhining Wang ${ }^{35}$, Liming Yang ${ }^{35}$, Jean Claude Zenklusen ${ }^{35}$

Tissue source sites: Analytical Biological Services Charles Saller ${ }^{38}$, Katherine $\mathrm{Tarvin}^{38}$; Fred Hutchinson Cancer Research Center Chu Chen ${ }^{39}$; Georgia Regents University Roni Bollag ${ }^{40}$, Paul Weinberger ${ }^{40}$; Greater Poland Cancer Centre Wojciech Golusiński ${ }^{29}$, Paweł Golusiński ${ }^{29}$, Matthew lbbs $^{29}$, Konstanty Korski ${ }^{29}$, Andrzej Mackiewicz ${ }^{29}$, Wiktoria Suchorska ${ }^{29}$, Bartosz Szybiak ${ }^{29}$, Maciej Wiznerowicz ${ }^{29}$; International Genomics Consortium Ken Burnett ${ }^{31}$, Erin Curley ${ }^{31}$, Johanna Gardner ${ }^{31}$, David Mallery ${ }^{31}$, Robert Penny ${ }^{31}$, Troy Shelton ${ }^{31}$, Peggy Yena ${ }^{31}$; Indiana University Simon Cancer Center Christina Beard ${ }^{41}$, Colleen Mitchell ${ }^{41}$, George Sandusky ${ }^{41}$; Johns Hopkins University Nishant Agrawal ${ }^{17}$, Julie Ahn ${ }^{17}$, Justin Bishop ${ }^{17}$, Joseph Califano ${ }^{17}$, Zubair Khan ${ }^{17}$; Princess Margaret Cancer Centre Jeff P. Bruce ${ }^{19}$, Angela B. Y. Hui ${ }^{19}$, Jonathan Irish ${ }^{27}$, Fei-Fei Liu ${ }^{27}$, Bayardo Perez-Ordonez ${ }^{28}$, John Waldron ${ }^{27}$; Ontario Institute for Cancer Research Paul C. Boutros ${ }^{18}$, Daryl M. Waggott ${ }^{18}$; The University of Texas MD Anderson Cancer Center Jeff Myers ${ }^{4}$, William N. William Jr; ; University of California San Diego Scott M. Lippman ${ }^{26}$; University of Miami Sophie Egea ${ }^{42}$, Carmen Gomez-Fernandez ${ }^{42}$, Lynn Herbert ${ }^{42}$; University of Michigan Carol R. Bradford ${ }^{20}$, Thomas E. Carey ${ }^{20}$, Douglas B. Chepeha $^{20}$, Andrea S. Haddad ${ }^{20}$, Tamara R. Jones ${ }^{20}$, Christine M. Komarck ${ }^{20}$, Mayya Malakh $^{20}$, Jonathan B. McHugh ${ }^{20}$, Jeffrey S. Moyer ${ }^{20}$, Ariane Nguyen ${ }^{20}$, Lisa A. Peterson $^{20}$, Mark E. Prince ${ }^{20}$, Laura S. Rozek ${ }^{20}$, Maureen A. Sartor ${ }^{20}$, Evan G. Taylor ${ }^{20}$ Heather M. Walline ${ }^{20}$, Gregory T. Wolf ${ }^{20}$; University of North Carolina at Chapel Hill Lori Boice ${ }^{9}$, Bhishamjit S. Chera ${ }^{9}$, William K. Funkhouser ${ }^{9}$, Margaret L. Gulley ${ }^{9}$, Trevor G. Hackman $^{9}$, D. Neil Hayes ${ }^{9}$, Michele C. Hayward ${ }^{9}$, Mei Huang ${ }^{9}$, W. Kimryn Rathmell', Ashley H. Salazar ${ }^{9}$, William W. Shockley ${ }^{9}$, Carol G. Shores ${ }^{9}$, Leigh Thorne ${ }^{9}$, Mark C. Weissler ${ }^{9}$, Sylvia Wrenn ${ }^{9}$, Adam M. Zanation ${ }^{9}$; University of Pittsburgh Simion I. Chiosea $^{22}$, Brenda Diergaarde 22 , Ann Marie Egloff ${ }^{22}$, Robert L. Ferris ${ }^{22}$, Marjorie Romkes $^{22}$, Raja Seethala ${ }^{22}$; Vanderbilt University Brandee T. Brown ${ }^{24}$, Yan Guo ${ }^{24}$, Michelle Pham ${ }^{24}$; Yale University Wendell G. Yarbrough ${ }^{30}$

${ }^{1}$ The Eli and Edythe L. Broad Institute of Massachusetts Institute of Technology and Harvard University, Cambridge, Massachusetts 02142, USA. ${ }^{2}$ Massachusetts General Hospital, 55 Fruit Street, Boston, Massachusetts 02114, USA. ${ }^{3}$ Canada's Michael Smith Genome Sciences Centre, BC Cancer Agency, Vancouver, British Columbia V5Z 4S6, Canada. ${ }^{4}$ The University of Texas MD Anderson Cancer Center, Houston, Texas 77030 USA. ${ }^{5}$ Harvard Medical School, Boston, Massachusetts 02115, USA. ${ }^{6}$ Brigham and Women's Hospital, Boston, Massachusetts 02115, USA. ${ }^{7}$ Boston Children's Hospital, Boston, Massachusetts 02115, USA. ${ }^{8}$ University of Kentucky, Lexington, Kentucky 40506 , USA. ${ }^{9}$ University of North Carolina at Chapel Hill, Chapel Hill, North Carolina 27599, USA. ${ }^{10}$ Dana-Farber Cancer Institute, 450 Brookline Avenue, Boston, Massachusetts 02215, USA. ${ }^{11}$ Harvard University, Boston, Massachusetts 02115, USA. ${ }^{12}$ Baylor College of Medicine, Houston, Texas 77030, USA. ${ }^{13}$ Memorial Sloan-Kettering Cancer Center, 1275 York Ave, New York, New York 10065, USA. ${ }^{14}$ University of California Santa Cruz, Santa Cruz, California 95064, USA. ${ }^{15}$ The Sidney Kimmel Comprehensive Cancer Center at Johns Hopkins University, Baltimore, Maryland 21287, USA. ${ }^{16}$ University of Southern California,1450 Biggy Street, NRT G511, Los Angeles, California 90033, USA. ${ }^{17}$ Johns Hopkins Medical Institutions, Baltimore, Maryland 21205, USA. ${ }^{18}$ Ontario Institute for Cancer Research 101 College Street, Suite 800, Toronto, Ontario M5G 0A3, Canada. ${ }^{19}$ Ontario Cancer Institute, Toronto, Ontario M5G 2M9, Canada. ${ }^{20}$ University of Michigan Comprehensive Cancer Center, Ann Arbor, Michigan 48109, USA. ${ }^{21}$ National Institute on Deafness and Other Communication Disorders, National Institutes of Health, Bethesda, Maryland 20892, USA. ${ }^{22}$ University of Pittsburgh, Pittsburgh, Pennsylvania 15213, USA ${ }^{23}$ The University of Chicago, Chicago, Illinois 60637, USA. ${ }^{24}$ Vanderbilt University Medical Center 7209 Medical Center East, South Tower Nashville, Tennessee 37232, USA.

${ }^{25}$ Albert Einstein College of Medicine, Bronx, New York 10461, USA. ${ }^{26}$ University of California San Diego, San Diego, California 92093, USA. ${ }^{27}$ University of Toronto, Toronto, Ontario M5G 2M9, Canada. ${ }^{28}$ University Health Network, Toronto, Ontario M5G 2C4, Canada. ${ }^{29}$ Greater Poland Cancer Centre, Garbary 15, 61-866, Poznań, Poland. ${ }^{30}$ Yale School of Medicine, 800 Howard Avenue, 4th Floor Yale Physicians Building, New Haven, Connecticut 06520-8041, USA. ${ }^{31}$ International Genomics Consortium, 445 North 5th Street, Phoenix, Arizona 85004, USA. ${ }^{32}$ The Research Institute at Nationwide Children's Hospital, Columbus, Ohio 43205, USA. ${ }^{33}$ SRA International, Fairfax, Virginia 22033, USA. ${ }^{34}$ Frederick National Laboratory for Cancer Research, 1050 Boyles Street, Frederick, Maryland 21702, USA. ${ }^{35}$ National Cancer Institute, 31 Center Drive 3A20, Bethesda, Maryland 20892, USA. ${ }^{36}$ Scimentis, LLC, Atlanta, Georgia 30666, USA. ${ }^{37}$ National Human Genome Research Institute, National Institutes of Health, Bethesda, Maryland 20892 . USA. ${ }^{38}$ Analytical Biological Services, Inc., 701 Cornell Drive, Wilmington, Delaware 19801, USA. ${ }^{39}$ Fred Hutchinson Cancer Research Center, Mailstop M5-C800, 1100 Fairview Avenue North, Seattle, Washington 98109, USA. ${ }^{40}$ Georgia Regents University, Augusta, Georgia 30912, USA. ${ }^{41}$ Indiana University Simon Cancer Center, Indianapolis, Indiana 46202, USA. ${ }^{42}$ University of Miami, Sylvester Comprehensive Cancer Center, 1550 NW 10th Avenue, Miami, Florida 33136, USA. 